\title{
A Rare Case of Subependymoma with an Atypical Presentation: A Case Report
}

\author{
Mohankumar Kurukumbia Amruta Muley ${ }^{\mathrm{b}} \quad$ Ganga Ramidi $^{\mathrm{b}}$ \\ Zakiya Wynn ${ }^{\mathrm{a}}$ Annapurni Jayam Trouth ${ }^{\mathrm{a}}$ \\ Departments of ${ }^{\mathrm{a}}$ Neurology and ${ }^{\mathrm{b}}$ Medicine, Howard University Hospital, Washington, \\ D.C., USA
}

\section{Key Words}

Intraventricular tumor · Subependymoma · Sensory dysesthesia

\begin{abstract}
A rare case of subependymoma in a young patient presenting with sensory dysesthesia is reported. Computed tomography scan and magnetic resonance imaging revealed a posterior fossa mass occluding the fourth ventricle with infiltration to the right side immediately behind the pontine tegmentum and impinging on the right spinothalamic tract. Postoperative tumor histopathology revealed the classical appearance of subependymoma. Subependymoma is a rare, asymptomatic, slow-growing, low-grade glioma of the central nervous system. If symptomatic, the clinical features are commonly secondary to hydrocephalus, but subependymoma presenting with sensory dysesthesia has never been reported in the literature.
\end{abstract}

\section{Introduction}

Subependymomas are uncommonly encountered ependymal tumors, accounting for less than $1 \%$ of all intracranial neoplasms and are distinguished from ordinary ependymomas by their overall better prognosis. Most of them are asymptomatic and detected incidentally at autopsy. It has been reported that there is $0.7 \%$ incidence of subependymoma among 1,000 patients with pathologically proven intracranial neoplasms [1]. When symptomatic, subependymomas often obstruct critical portions of the cerebrospinal fluid pathway, causing hydrocephalus. The most common symptoms include headache, gait ataxia, vertigo or dizziness, nausea and vomiting [2]. Here, we present a case of symptomatic subependymoma with a very rare clinical presentation. 


\section{Case Presentation}

A 19-year-old male presented to us with complaints of pain and numbness of 1 month's duration on the left side of his body, including the face and the upper and lower extremities. He reported no significant medical antecedent history. This complaint was preceded by herpes zoster involving the V3 distribution of the trigeminal nerve. The lesions completely disappeared after treatment, but the pain and numbness persisted. He was treated with Pregabalin for the pain without any response. Clinical examination revealed decreased perception of pain and touch involving the whole left side of his body. The rest of the detailed neurological examination was normal.

With the suspicion of central pathology, magnetic resonance imaging (MRI) of the brain with gadolinium was carried out which revealed presence of a posterior fossa mass (fig. 1, fig. 2). The mass was almost occluding the fourth ventricle with infiltration to the right side immediately behind the pontine tegmentum and probably impinging on the right spinothalamic tract causing the presenting symptoms. There was a central area of calcification and mild enhancement to the contrast. The patient underwent craniotomy and complete macroscopic removal of the tumor. Postoperative tumor histopathology revealed the classical appearance of subependymoma with characteristic clusters of nuclei in a dense fibrillary background (fig. 3). Mitotic activity, vascular proliferation or necrosis was not observed. Absence of perivascular pseudorosettes or true rosettes excluded the possibility of ependymoma. In the postoperative period, the patient remained pain free and no radiological evidence of recurrence was seen at follow-up after 1 year.

Written informed consent was obtained from the patient for publication of this case report and accompanying images.

\section{Discussion}

Subependymoma is a rare, benign, slow-growing neoplasm that was first described by Scheinker [3] in 1945 as a tumor arising from the subependymal cell plate.

Subependymomas are most commonly located in the fourth and lateral ventricles, but have also been reported arising in the third ventricle, septum pellucidum, and spinal canal. Since its original description, more than 100 case reports of intracranial subependymomas have been described. Due to its rarity and variable imaging characteristics, reliable preoperative diagnosis remains challenging [4].

Most subependymomas are smaller than $2 \mathrm{~cm}$ in diameter. However, symptomatic subependymomas are usually larger, averaging about $3-5 \mathrm{~cm}$ in greatest dimension $[5,6]$. The clinical presentation is nonspecific. Most symptomatic patients (80\%) present with symptoms related to hydrocephalus as a consequence of ventricular obstruction [7]. Less commonly, focal neurological deficits (27\%), seizures (9\%), and subarachnoid hemorrhage (4.5\%) have been reported [7]. Males are more commonly affected and most reported cases (82\%) have occurred in patients older than 15 years [7]. At least half of the reported cases have occurred in the fourth ventricle, with most of the remainder arising in the lateral ventricle [5]. Jooma et al. [8] reported a series of 12 cases of fourth ventricle subependymoma. The commonest symptoms were loss of balance (9/12) and vertigo $(8 / 12)$. Nystagmus was the commonest sign (11/12). None of the cases presented with sensory dysesthesia.

Commonly, patients with subependymomas have long duration and slow progression of symptoms. They usually present in the fifth or sixth decade of life [8]. In our case, striking features were short duration of atypical symptoms and early age of presentation. 
Subependymomas have a white to grayish color and are well circumscribed with a firm texture [7]. The tumors grow in a slow deliberate fashion, are usually avascular, and are attached to the ventricular wall by a narrow pedicle [7]. Although the exact histogenesis is still uncertain, they most likely arise from subependymal glial cells. Other possible sites include astrocytes from the subependymal plate, ependymal cells, and a mixture of ependymal and astrocytic cells. In our case, characteristic clusters of nuclei in a dense fibrillary background were observed.

Common imaging characteristics of subependymoma include a lobulated, well-defined intraventricular mass with no paraventricular extension $[7,9,10]$. The majority of subependymomas are solid or solid with areas of cystic degeneration [1,7]. Calcification is common, occurring in $32-50 \%$ of cases $[7,8]$. Computed tomography (CT) enhancement characteristics of subependymoma are variable ranging from absent to marked enhancement $[1,7,9,10]$. In our case, there was central calcification, minimal enhancement to contrast and paraventricular extension.

MRI characteristics commonly include a well-defined solid or mixed solid and cystic intraventricular mass. The solid component is hypo- or isointense on T1-weighted images and hyperintense on T2-weighted images $[1,7,9,10]$. The heterogeneous signal commonly seen in subependymomas correlates well with histopathologic findings, including necrosis, calcification, microcystic change, and hemorrhage [11]. Peritumoral edema is an infrequent feature. Similar to CT, MRI enhancement characteristics are variable, including absent to intense enhancement $[7,11]$. MRI has proven most useful in defining the size and extent of the tumor, as well as its relationship to other anatomical structures, such as vasculature, playing a critical role in preoperative planning [8].

Included in the differential diagnosis of fourth ventricular masses are ependymoma, subependymoma, choroid plexus papilloma, metastasis, rarely meningioma, central neurocytoma and subependymal giant cell astrocytcoma. Age, location and imaging features are helpful in narrowing the number of differential diagnoses.

In attempting to differentiate subependymomas from ependymomas with imaging studies alone, Lobato et al. [7] noted that subependymomas tend to be intraventricular, whereas ependymomas tend be paraventricular. They also reported that hyperattenuation compared with brain parenchyma, enhancement, calcification, and cyst formation was also more commonly seen in ependymomas than in subependymomas. However, none of these features are sufficiently pronounced to be pathognomonic for either lesion. Like in our case, the tumor was paraventricular, which raised the suspicion in favor of ependymoma preoperatively. Therefore, the distinctions between these tumors are even less apparent when they arise in the fourth ventricle [7].

Choroid plexus papillomas most commonly occur in young patients and intensely enhance after contrast administration [4]. Central neurocytomas are similar to subependymomas in their cystic appearance but are usually isointense with gray matter on both T1- and T2-weighted sequences, whereas subependymomas are almost always hyperintense on T2-weighted images [7].

Subependymal giant cell astrocytomas always occur near the foramen of Monro, calcify frequently, enhance intensely, and are often associated with ventricular wall calcification $[4,7]$. Similarly, meningiomas frequently calcify and intensely enhance. Metastasis and malignant gliomas are more likely to grow rapidly and often lack calcification [8]. 
Optimal therapy for symptomatic subependymomas is complete surgical excision. This can often be achieved with supratentorial subependymomas, given that these tumors are usually well demarcated, avascular, and noninvasive $[1,7,10]$. Fourth ventricle subependymomas, however, frequently arise from the floor of the fourth ventricle and the likelihood of cranial nerve injury often precludes complete surgical resection $[1,8,10]$. Perioperative mortality rates as high as $42 \%$ have been reported with surgical resection of fourth ventricle subependymomas [8]. In cases of symptomatic fourth ventricle subependymomas where complete resection cannot be achieved, surgery should be directed towards debulking and improving CSF outflow $[8,10]$.

Most authors do not advocate postoperative radiation therapy after complete surgical resection of pure subependymoma as there is a good prognosis with surgery alone $[7,9$, 10]. Lombardi et al. [10] reported follow-up data in 21 patients who underwent surgery for subependymoma and found that, of the 19 patients who survived the perioperative period, 12 underwent gross total resection with no evidence of tumor recurrence or tumor-related death. The remaining 7 patients were treated with radiation therapy and follow-up imaging demonstrated a greater radiographic response at doses of 5,000 cGy or greater, supporting an argument for radiation therapy in patients with residual tumor or evidence or tumor progression [10]. In our case, the tumor was well localized with minimal paraventricular spread and without cranial nerves involvement, which influenced the better surgical outcome.

\section{Conclusion}

We conclude that subependymomas are rare, often asymptomatic masses related to the ventricular system. Intraventricular subependymomas have some distinguishing clinical features as well as MRI and CT characteristics, but none of them is pathognomic. The best treatment for subependymomas is total resection and, if this is not possible, debulking of the tumor followed by radiation therapy can be considered. Diagnosis of subependymoma can be easily missed in view of such atypical presentation. A high index of suspicion is required to clinch the diagnosis early in the process because of its excellent prognosis with timely treatment.

\section{Disclosure Statement}

The authors declare that they have no competing interests. 


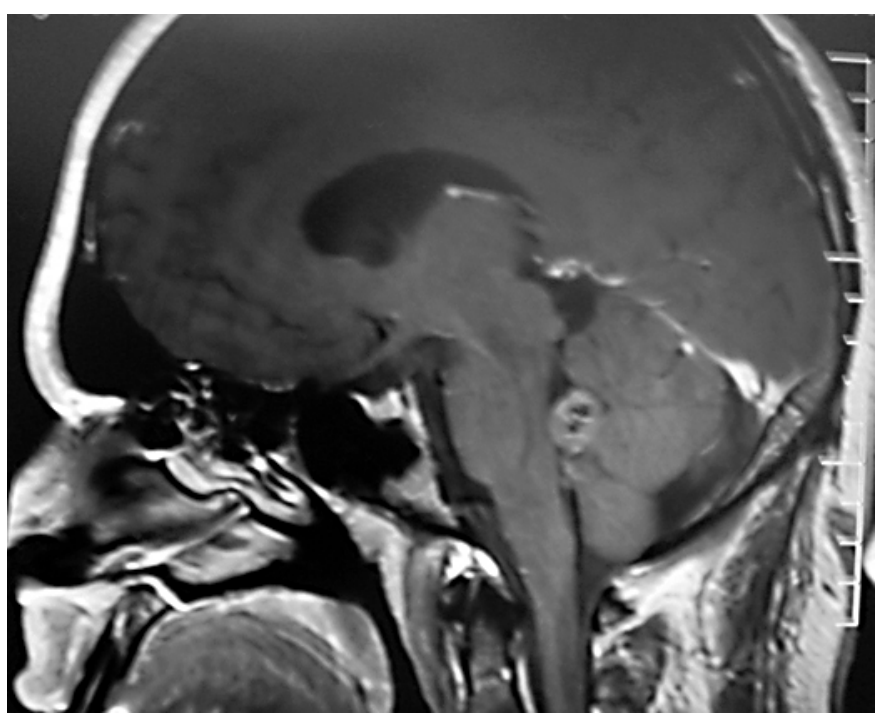

Fig. 1. MRI of the brain with gadolinium (sagittal section). Mass in the fourth ventricle with minimum enhancement and central calcification (confirmed by CT of the head).

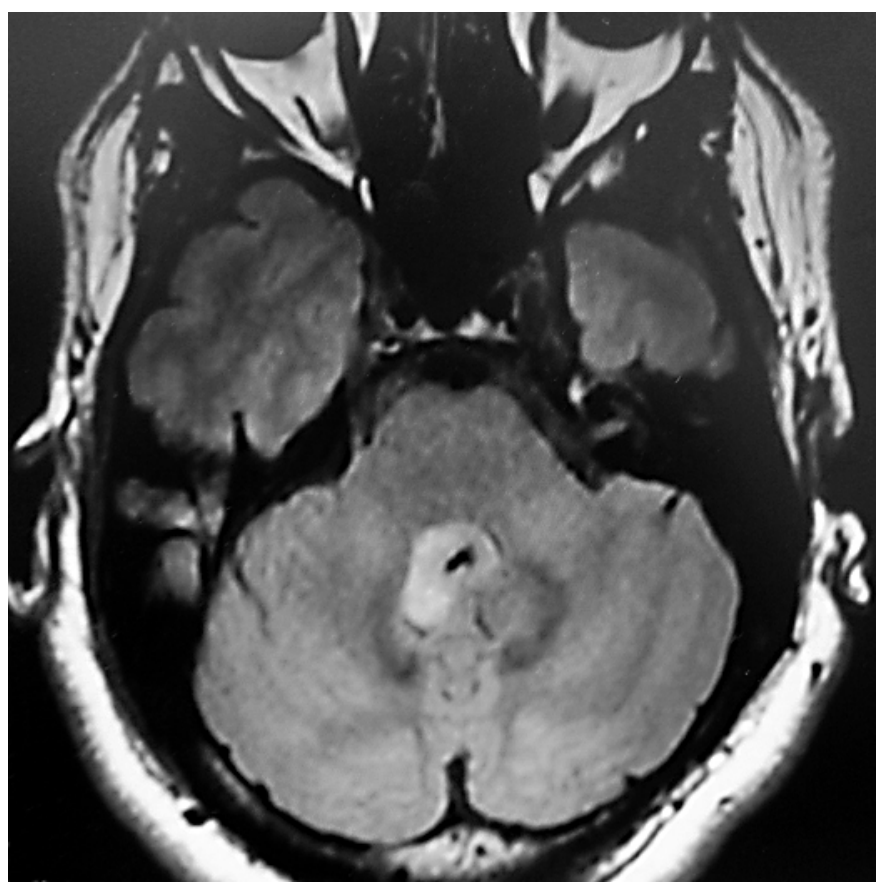

Fig. 2. MRI of the brain (axial section). Mass in the fourth ventricle with minimal paraventricular spread. 


\begin{tabular}{|c|c|c|c|}
\hline $\begin{array}{l}\text { ase Reports in } \\
\text { Neurology }\end{array}$ & $\begin{array}{l}\text { Case Rep Neurol 2011;3:227-232 } \\
\text { DOI: } 10.1159 / 000333061\end{array}$ & $\begin{array}{l}\text { Published online: } \\
\text { October 1, } 2011\end{array}$ & $\begin{array}{l}\text { @ } 2011 \text { S. Karger AG, Basel } \\
\text { ISSN 1662-680X } \\
\text { www.karger.com/crn }\end{array}$ \\
\hline
\end{tabular}

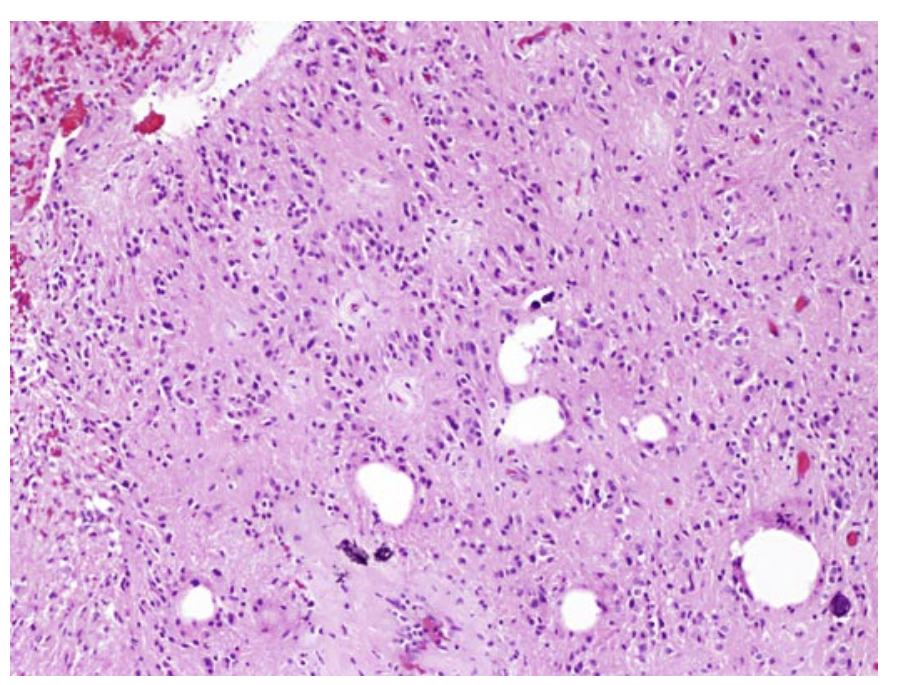

Fig. 3. Postoperative histopathology of the mass showing characteristic clusters of nuclei in a dense fibrillary background suggestive of subependymoma.

\section{References}

1 Matsumara A, Ahyai A, Hori A, Schaake T: Intracerebral subependymomas: clinical and neuropathological analyses with special reference to the possible existence of a less benign variant. Acta Neurochir 1989;96:15-25.

$\checkmark 2$ Prayson RA, Suh JH: Subependymomas: clinicopathologic study of 14 tumors, including comparative MIB-1 immunohistochemical analysis with other ependymal neoplasms. Arch Pathol Lab Med 1999;123:306-309.

3 Scheinker I: Subependymoma: a newly recognized tumor of subependymal derivation. J Neurosurg 1945;2:232-240.

-4 Koeller KK, Sandberg GD: Cerebral intraventricular neoplasms: radiologic-pathologic correlation. Radiographics 2002;22:1473-1505.

5 Scheithauer B: Symptomatic subependymoma: report of 21 cases with review of the literature. J Neurosurg 1978;49:689-696.

6 Chiechi M, Smirniotopoulos J, Jones R: Intracranial subependymomas: CT and MR imaging features in 24 cases. AJR Am J Roentgenol 1995;165:1245-1250.

7 Lobato R, Sarabia M, Castro S: Symptomatic subependymoma: report of four new cases studied with computed tomography and review of the literature. Neurosurgery 1986;19:594-598.

8 Jooma R, Torrens MJ, Bradshaw J, Brownell B: Subependymomas of the fourth ventricle: surgical treatment in 12 cases. J Neurosurg 1985;62:508-512.

9 Im SH, Paek SH, Choi YL, Chi JG, Kim DG, Jung HW, Cho BK: Clinicopathological study of seven cases of symptomatic supratentorial subependymoma. J Neurooncol 2003;61:57-67.

10 Lombardi D, Scheithauer BW, Meyer FB, Forbes GS, Shaw EG, Gibney DJ, Katzmann JA: Symptomatic subependymoma: a clinicopathological and flow cytometric study. J Neurosurg 1995;75:583-588.

11 Hoeffel C, Boukobza M, Polivka M, Lot G, Guichard JP, Lafitte F, Reizine D, Merland JJ: MR manifestations of subependymomas. Am J Neuroradiol 1995;16:2121-2129. 